\title{
Noise-Induced Transitions in a Population Growth Model Based on Size-Dependent Carrying Capacity
}

\author{
Neeme Lumi, Ain Ainsaar, and Romi Mankin \\ Institute of Mathematics and Natural Sciences, Tallinn University, Narva Road 25, 10120 Tallinn, Estonia \\ Correspondence should be addressed to Neeme Lumi; neeme.lumi@tlu.ee
}

Received 3 December 2013; Revised 14 January 2014; Accepted 26 January 2014; Published 4 March 2014

Academic Editor: Hao Shen

Copyright ( 2014 Neeme Lumi et al. This is an open access article distributed under the Creative Commons Attribution License, which permits unrestricted use, distribution, and reproduction in any medium, provided the original work is properly cited.

\begin{abstract}
The stochastic dynamics of a population growth model with size-dependent carrying capacity is considered. The effect of a fluctuating environment on population growth is modeled as a multiplicative dichotomous noise. At intermediate values of population size the deterministic counterpart of the model behaves similarly to the Von Foerster model for human population, but at small and very large values of population size substantial differences occur. In the stochastic case, an exact analytical solution for the stationary probability distribution is found. It is established that variation of noise correlation time can cause noiseinduced transitions between three different states of the system characterized by qualitatively different behaviors of the probability distributions of the population size. Also, it is shown that, in some regions of the system parameters, variation of the amplitude of environmental fluctuations can induce single unidirectional abrupt transitions of the mean population size.
\end{abstract}

\section{Introduction}

The dynamical stability of populations and ecosystems is known to govern their responsiveness to fluctuating environmental conditions, determining with what reliability natural resources provide life-sustaining services to society. Therefore, population and ecosystem dynamics is a major structuring theme in ecology [1]. The most productive abstraction of a self-regulation mechanism for population growth is the Malthus-Verhulst (logistic) model with a constant carrying capacity, which (or its various modifications like Richards model, Gompertz model, etc.) describes well a large class of populations [2, 3]. However, for some populations, such as the human one, empirical data show that the logistictype models are irrelevant to describe the growth process [4-6]. For example, the historical estimates for the past 2000 years of human population growth indicate that human numbers have been growing faster than exponentially for most of the known history [4, 5]. In 1960 Von Foerster et al. suggested that the classical logistic growth in the case of the human population should be changed, because the individuals in the system can form coalitions that, due to technological progress, can grow faster [4]. In the light of the empirical data and the positive feedback mechanism between innovation and population size, in [4-7] the dynamics of human community was modeled by a growth model with a size-dependent "carrying capacity" K (called Von Foerster model), assuming a simple power-law relationship $K \sim x^{1+\alpha}$, where $\alpha>0$ and $x$ is the population size. Although this model fits the population data for humans during the past 2000 years quite well, in an evolutionary time scale it seems, however, that for the growth of an initially small human population a logistic model is more appropriate. Moreover, as the growth of any expanding population must eventually be limited by a shortage of resources and habitats, for very large values of the population size the Von Foerster model should be modified. Ecological investigations indicate that population dynamics is sensitive to fluctuations of environmental parameters (noise) $[8,9]$. Noise, via its interaction with nonlinearity in physical, chemical, and biological systems, has given rise to several counterintuitive phenomena: stochastic resonance $[10-12]$, noise-enhanced stability $[13,14]$, stochastic transport in ratchets $[15,16]$, noise-induced phase transitions [1720], noise-induced transitions [21, 22], memory generated resonance [23-25], and so forth. In particular, the authors of [26] have shown that, in some symbiotic ecosystems, colored 
fluctuations of the carrying capacities of populations can induce bistability and produce abrupt changes between stable states. The fact that external multiplicative noise can induce multistability as well as discontinuous transitions in some complex systems (see, e.g., $[27,28]$ ) inspired us to apply an analogous approach to analyze single population models. It is of interest, both from theoretical and practical viewpoints, to know whether environmental-noise-induced transitions and abrupt changes of mean population sizes also occur in population growth models with a size-dependent carrying capacity.

Thus motivated, we consider a stochastic population growth model with a size-dependent carrying capacity $K$. For the abovementioned reasons, the dependence of $K$ on the population size $x$ is assumed such that for small values of $x$ the model behaves similarly to a logistic model; for intermediate and large values of $x$ it behaves like Von Foerster model, and for very large values of population size the carrying capacity tends to a finite constant value. The influence of fluctuating environmental parameters on the growth of the population is modeled as colored fluctuations of the carrying capacity. For the sake of mathematical simplicity it is practicable to use such types of colored noise that enable evaluation of stationary probability density of the population size exactly for any value of the noise correlation time. The corresponding analytical solution would be of major interest in itself, since it can serve as a standard situation for comparison with approximate solutions and/or numerical simulations in the more realistic cases of bounded colored noises. The simplest noise of this kind is the symmetric dichotomous Markovian noise, also known as the random telegraph signal [29]. Thus motivated, in this work the fluctuations of the carrying capacity are modeled as dichotomous Markovian noise.

The main purpose of this paper is to provide exact formulas for the analytical treatment of the stationary probability density of the population size. Moreover, we will demonstrate the presence of noise-induced transitions of the probability distribution and single unidirectional abrupt transitions for the mean population size in the considered population growth model.

To avoid misunderstanding, let us mention that we use the term "noise-induced transitions" in the sense of $[17,22]$, meaning that this corresponds to a qualitative change in the probability law characterizing the random variable in response to noise parameters. The number and position of the extrema of the stationary probability density are, from this point of view, merely a practical way to monitor such a qualitative change.

\section{Deterministic Population Growth Model}

As was mentioned in the introduction, the present model is based on a generalization of the logistic equation for population growth (cf. $[5,7]$ )

$$
\frac{d x(t)}{d t}=r x[K(x)-x(t)]
$$

where $x(t)$ is the population size at time $t$ and the constant $r$ is the growth rate parameter. Note that in the case of a constant carrying capacity $K$ this equation is the same as the usual logistic equation [2]

$$
\frac{d x(t)}{d t}=\tilde{r} x\left(1-\frac{x(t)}{K}\right), \quad \tilde{r}=r K .
$$

In this work the carrying capacity $K(x)$ is assumed to be of the form

$$
K(x)=K_{0}+\frac{\epsilon x^{1+\alpha}}{1+\epsilon \gamma x^{1+\alpha}}
$$

where $K_{0}$ (henceforth, natural carrying capacity) is the carrying capacity without any influence of the population on the value of $K$. For example, for the human population the exponent $\alpha>0$ and the parameter $\epsilon>0$ characterize an increase of the carrying capacity due to the technological progress such as the use of tools and fire, the development of agriculture, and the introduction of fossil fuels and fertilizers as well as expansion into new habitats and the removal of limiting factors by the development of vaccines, pesticides, antibiotics, and so forth $[5,6]$. The saturation parameter $\gamma>0$ takes into account that the growth of any expanding population must eventually be limited by a shortage of resources and habitats.

In the rest of the paper, we will always assume that the parameters $\alpha, \epsilon, K_{0}$, and $\gamma$ are restricted to the inequalities

$$
0<\alpha<1, \quad \gamma \ll \epsilon^{1 / \alpha}, \quad \gamma \ll K_{0}^{-1}
$$

These conditions seem to be relevant for the human population as we bear in mind several datasets expressing the development of mankind on Earth in terms of size and economic impact represented in [5]. For the sake of simplicity, we assume that $\alpha$ is of the form $\alpha=m / n$, where $n>m>0$ are integers and $m / n$ is irreducible (i.e., not equal to some other $k / s$, where $k<m$ and $s<n$ are integers).

By applying the change of variables

$$
y=x^{1 / n}
$$

we rewrite (1) with (3) as

$$
\frac{d y}{d t}=\frac{r \gamma \epsilon y}{n\left(1+\gamma \epsilon y^{n+m}\right)} F(y), \quad y \geq 0,
$$

where

$$
\begin{aligned}
F(y)= & -y^{2 n+m}+\left(\frac{1}{\gamma}+K_{0}\right) y^{n+m} \\
& -\frac{1}{\gamma \epsilon} y^{n}+\frac{K_{0}}{\gamma \epsilon} .
\end{aligned}
$$

Depending on the natural carrying capacity $K_{0}$ the solution of (6) has either four or two equilibria. The unstable trivial equilibrium, $y=0$, occurs for all biologically possible values of the system parameters. 


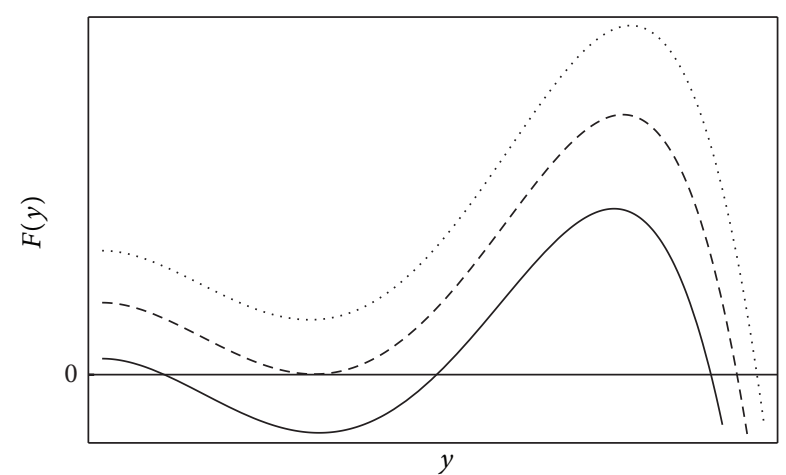

Figure 1: Sketch of the typical dependencies of the polynomial $F$ versus $y$ by conditions (4) at various values of the natural carrying capacity $K_{0}$ (see also (7)). Solid line, $K_{0}<K_{0 c}$; dashed line, $K_{0}=K_{0 c}$; dotted line, $K_{0}>K_{0 c}$.

If the natural carrying capacity is below a critical value $K_{0 c}$, there are, in addition to the trivial equilibrium, two stable and one unstable equilibria, but if $K_{0}>K_{0 c}$, only one nontrivial stable equilibrium occurs. This fact is illustrated in Figure 1 with dependencies of the polynomial $F(y)$ on variable $y$ at various values of $K_{0}$. By conditions (4) the critical carrying capacity $K_{0 c}$ is approximately given by the equation

$$
K_{0 c} \approx \frac{\alpha}{\epsilon^{1 / \alpha}}\left(\frac{1}{1+\alpha}\right)^{1+1 / \alpha}
$$

It is notable that in the case $K_{0}<K_{0 c}$ an explosive increase of the population size to a very large limit value $\left(x_{\max } \sim 1 / \gamma\right)$ is not possible if the initial population size $x(0)$ is lower than the nontrivial unstable equilibrium value. In this case $x$ saturates at the smaller stable equilibrium point, which is of the same size order as the natural carrying capacity $K_{0}$. This circumstance and (8) give an ecological interpretation of the parameter $\epsilon$. The quantity $\epsilon^{-1 / \alpha}$ is a measure of the typical value of the population size above which an explosive increase of the population size is possible. This interpretation is in accordance with intuition: a significant growth of a population due to technological progress is possible only if the population is large enough.

\section{Stochastic Model}

Random interaction with the environment (climate, diseases, etc.) is taken into account by introducing a colored noise in $K(x)$. From now on we will use fluctuations of the natural carrying capacity

$$
K_{0}=\widetilde{K}_{0}(1+a Z(t))
$$

where colored noise $Z(t)$ is assumed to be a dichotomous Markovian stochastic process [29]. The dichotomous process is a random stationary Markovian process consisting of jumps between two values $z=-1,1$. The jumps follow in time according to a Poisson process, while the values occur with the stationary probability $1 / 2$. The mean value of $Z(t)$ and the correlation function are

$$
\langle Z(t)\rangle=0, \quad\left\langle Z(t) Z\left(t^{\prime}\right)\right\rangle=e^{-v\left|t-t^{\prime}\right|},
$$

where the switching rate $v$ is the reciprocal of the noise correlation time $v=1 / \tau_{c}$. Obviously, model (1) with (3) and (9) is biologically meaningful only if

$$
a<1
$$

that is, the natural carrying capacity is nonnegative. To proceed further with the analytical examination of the stochastic version of model (1) it is reasonable to introduce the following notations:

$$
\begin{gathered}
K_{01}=\widetilde{K}_{0}(1+a), \quad K_{02}=\widetilde{K}_{0}(1-a), \\
F_{j}(y)=-y^{2 n+m}+\left(\frac{1}{\gamma}+K_{0 j}\right) y^{n+m} \\
-\frac{1}{\gamma \epsilon} y^{n}+\frac{K_{0 j}}{\gamma \epsilon}, \quad j=1,2 .
\end{gathered}
$$

Now the stationary behavior of the stochastic system described with (6), (7), and (9) can be analytically studied by means of the corresponding composite master equation

$$
\begin{aligned}
\frac{\partial P_{j}(y, t)}{\partial t}= & -\frac{\partial}{\partial y}\left[\frac{r \gamma \epsilon y}{n\left(1+\gamma \epsilon y^{n+m}\right)} F_{j}(y) P_{j}(y, t)\right] \\
& +\sum_{k=1}^{2} U_{j k} P_{k}(y, t)
\end{aligned}
$$

where $P_{j}(y, t)$ denotes the probability density for the combined process $\left(y, z_{j}, t\right) ; j, k=1,2 ;-z_{2}=z_{1}=1$; and

$$
\left(U_{j k}\right)=v\left(\begin{array}{cc}
-\frac{1}{2} & \frac{1}{2} \\
\frac{1}{2} & -\frac{1}{2}
\end{array}\right) .
$$

The stationary probability density in the $y$ space, $P(y)$, is then evaluated via the stationary probability densities $P_{j}^{s}(y)$ for the states $\left(y, z_{j}\right)$ :

$$
P(y)=P_{1}^{s}(y)+P_{2}^{s}(y) .
$$

To establish the boundaries of the stationary process $y(t)$ we consider the deterministic equation (6), where the natural carrying capacity $K_{0}$ is replaced by $K_{0 j}$. Two cases should be discerned (cf. also Figure 1): (i) for $K_{01}>K_{0 c}$ (see (8)) there is just one positive solution of the polynomial equation

$$
F_{1}(y)=0 \text {, }
$$

which is the stable fixed point $y=y_{1}$ of the deterministic equation (6) at the noise value $z=1$. Although at the noise value $z=-1$ (6) may have, depending on the value of $K_{02}$, two (or one) stable fixed points, in the long time limit (i.e., in 


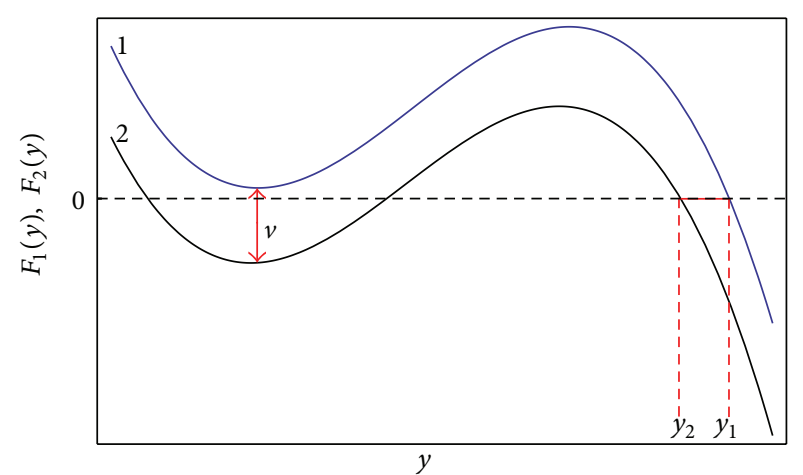

(a)

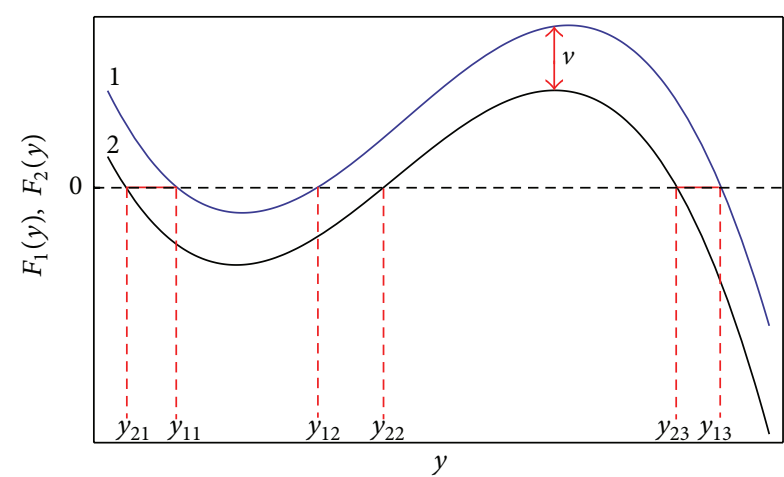

(b)

FIgURE 2: A schematic plot of the dependence of the polynomials $F_{1}$ and $F_{2}$ on $y$ and attracting intervals. Because of the influence of the noise $Z(t)$ in (6), (7), and (9) the dependence of the function $F(y)$ on $y$ fluctuates with a switching rate $v$ between two forms $F_{1}(y)$ and $F_{2}(y)$, which correspond to noise values $z=1$ and $z=-1$ and are illustrated by curves 1 and 2, respectively. The bold lines depict the attracting intervals. (a) The case of $K_{01}>K_{0 c}$; the stable fixed points of (6) are denoted by $y_{1}$ and $y_{2}$. (b) The case of $K_{01}<K_{0 c}$; the fixed points for (6) by noise states $z=1$ and $z=-1$ are $y_{1 i}$ and $y_{2 i}, i=1,2,3$, respectively.

a stationary regime) because of the switching of noise values the greater fixed point $y=y_{2}$ is eventually realized (cf. also Figure 2(a)). Thus, in this case all trajectories $y(t)$ in a stationary regime satisfy the following inequalities:

$$
y_{1} \geq y(t) \geq y_{2} .
$$

(ii) In the case of $K_{01}<K_{0 c}$ the polynomial equation (16) has three positive solutions of $y_{11}<y_{12}<y_{13}$, of which $y_{11}$ and $y_{13}$ are the stable fixed points of (6) with $z=1$. Obviously, in the noise phase $z=-1$ (6) also has two stable fixed points, $y=y_{21}$ and $y=y_{23}$. Therefore, depending on an initial value $y(0)$, we can single out the following alternatives for a stationary regime (cf. also Figure 2(b)).

(1) If $y(0)<y_{12}$, then the trajectories $y(t)$ are restricted in the interval

$$
y_{11} \geq y(t) \geq y_{21} .
$$

(2) For $y(0)>y_{22}$ it follows that

$$
y_{13} \geq y(t) \geq y_{23} .
$$

(3) In the case of $y_{12}<y(0)<y_{22}$ both attracting intervals (18) and (19) are possible; that is, with probabilities $w$ and $1-w$ we can find the trajectories $y(t)$ in the intervals (18) and (19), respectively.

\section{Stationary Probability Distribution}

For a stationary state we can solve (13), taking as the boundary condition that there is no probability current at the boundary (17) (for the case of (18) and (19), the results are analogous). After quite simple calculations one can find

$$
\begin{aligned}
P(y)= & \frac{C\left(1+\gamma \epsilon y^{n+m}\right)^{2}}{y F_{1}(y) F_{2}(y)} \\
& \times \exp \left\{-\frac{\nu n}{r \gamma \epsilon} \int_{y_{2}}^{y} \frac{F(\xi)\left(1+\epsilon \gamma \xi^{n+m}\right)}{\xi F_{1}(\xi) F_{2}(\xi)} d \xi\right\},
\end{aligned}
$$

where the constant $C$ is determined by the normalization condition

$$
\int_{y_{2}}^{y_{1}} P(y) d y=1 .
$$

Taking into account the transformation (5), it is easy to establish that for the original variable $x$ the stationary probability density $\widetilde{P}(x)$ can be written as

$$
\widetilde{P}(x)=\frac{y(x)}{n x} P(y(x)) .
$$

In the following, we assume that all $2 n+m$ zeros of the polynomials $F_{j}(y)$ are distinct; that is, we assume that $K_{0 j} \neq K_{0 c}$. In this case it is convenient to use the partial fraction expansion

$$
\begin{aligned}
\frac{1}{y F_{j}(y)}= & \frac{1}{y F_{j}(0)}+\left[F_{j}^{\prime}\left(y_{j}\right)\right]^{-1} \frac{1}{\left(y-y_{j}\right) y_{j}} \\
& +\sum_{k=1}^{2 n+m-1}\left[F_{j}^{\prime}\left(y_{j}^{(k)}\right)\right]^{-1} \frac{1}{\left(y-y_{j}^{(k)}\right) y_{j}^{(k)}},
\end{aligned}
$$

where $F_{j}^{\prime}(y) \equiv d F_{j}(y) / d y$ and $y_{j}^{(k)}$ are zeros of the polynomial $F_{j}(y)$, which are distinct from $y_{j}\left(y_{j}\right.$ is also a zero of $\left.F_{j}(y)\right)$. By denoting

$$
G_{j}(y):=\sum_{k=1}^{2 n+m-1}\left[F_{j}^{\prime}\left(y_{j}^{(k)}\right)\right]^{-1} \frac{1}{\left(y-y_{j}^{(k)}\right) y_{j}^{(k)}},
$$

one can get

$$
\begin{array}{r}
\widetilde{P}(x)=\frac{\widetilde{C} M(x)\left(1+\gamma \epsilon x^{1+\alpha}\right)^{2}}{x^{1+\gamma / r} \widetilde{K}_{0}\left(1-a^{2}\right)\left|x^{1 / n}-y_{1}\right|^{1-A_{1}}\left|x^{1 / n}-y_{2}\right|^{1-A_{2}}}, \\
x \in\left(y_{2}^{n}, y_{1}^{n}\right),
\end{array}
$$




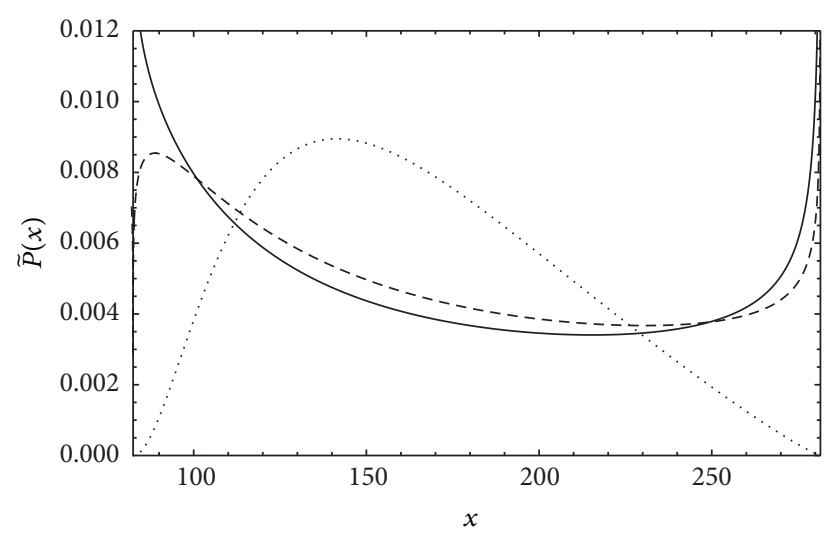

(a)

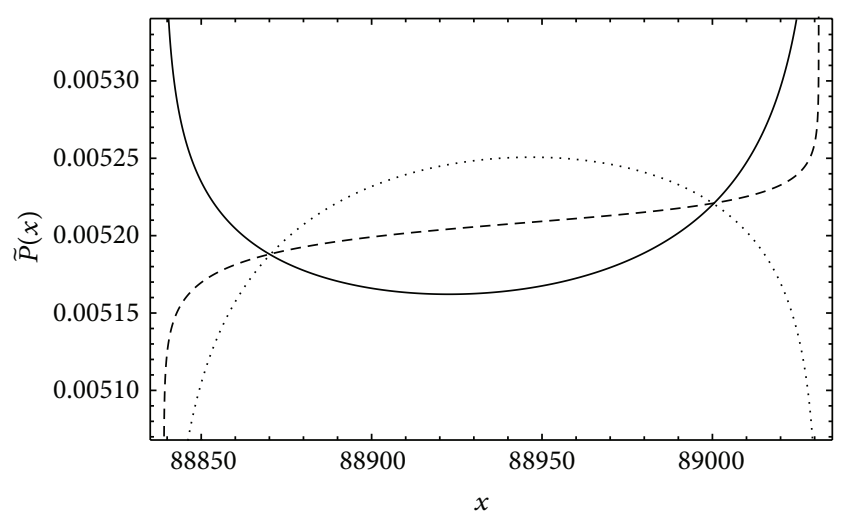

(b)

FIgURE 3: The stationary probability density $\widetilde{P}(x)$ versus the population size $x$, computed from (25)-(27). The system parameter values are $\widetilde{K}_{0}=100, \epsilon=0.03, \gamma=10^{-5}$, and $\alpha=0.5$. (a) The case of $K_{01}<K_{0 c}$ with (18), $a=0.4$. Solid line, $v=45$; dashed line, $v=55$; dotted line, $v=150$. (b) The case of $K_{01}>K_{0 c}, a=0.8$. Solid line, $v=73000$; dashed line, $v=74000$; dotted line, $v=75000$.

where

$$
\begin{aligned}
& M(x)=\prod_{j=1}^{2}\left\{\frac{1}{\prod_{k=1}^{2 n+m-1}\left(x^{1 / n}-y_{j}^{(k)}\right)}\right. \\
& \times \exp \left[-\frac{v n}{2 r \gamma \epsilon}\right. \\
& \quad \times \int_{y_{2}}^{x^{1 / n}}\left(G_{j}(\xi)\left(1+\epsilon \gamma \xi^{n+m}\right)\right. \\
&\left.\left.\left.\quad+\frac{\epsilon \gamma\left(\xi^{n+m-1}-y_{j}^{n+m-1}\right)}{\left(\xi-y_{j}\right) F_{j}^{\prime}\left(y_{j}\right)}\right) d \xi\right]\right\},
\end{aligned}
$$

$$
A_{j}=\frac{\nu n\left(1+\epsilon \gamma y_{j}^{n+m}\right)}{2 r \gamma \epsilon y_{j}\left|F_{j}^{\prime}\left(y_{j}\right)\right|}, \quad j=1,2,
$$

and $\widetilde{C}$ is the normalization constant. It should be noted that the function $M(x)$ is bounded in the interval $x \in\left[y_{2}^{n}, y_{1}^{n}\right]$ including also the boundary points $y_{2}^{n}$ and $y_{1}^{n}$. Formulas (25)(27) are also applicable for the attracting intervals represented by inequalities (18) and (19). In this case $y_{2}$ and $y_{1}$ in (25)-(27) should be replaced by $y_{21}$ and $y_{11}$ (or by $y_{23}$ and $y_{13}$ for (19)), respectively.

In Figure 3, the probability density $\widetilde{P}(x)$ is plotted as a function of the population size for various values of the noise switching rate $\nu$. An important observation here is that three types of curves are distinguishable in Figure 3, namely, Ushaped ones, bell-shaped ones, and curves with either two or no local extrema. We interpret these three qualitatively different shapes of $\widetilde{P}(x)$ as different states of the stationary system (see also $[17,22])$. From (25) and (27) it follows that the critical noise switching rates $v_{c j}, j=1,2$, at which noiseinduced transitions occur can be expressed as

$$
v_{c j}=\frac{2 r \gamma \epsilon y_{j}\left|F_{j}^{\prime}\left(y_{j}\right)\right|}{n\left(1+\gamma \epsilon y_{j}^{n+m}\right)}, \quad j=1,2 .
$$

As the noise switching rate $v$ increases (or noise correlation time $\tau_{c}=1 / \nu$ decreases) the system undergoes two transitions: for $v<v_{c 2}$, the probability density exhibits a Ushaped form; if $v_{c 2}<v<v_{c 1}$, then $\widetilde{P}(x)$ is characterized with two or no local extrema; finally, in the case of $v>v_{c 1}$, $\widetilde{P}(x)$ approaches a bell-shaped form. So the value of noise correlation time has a crucial influence on the position of the most probable population size in the attracting intervals. It should be noted that although in the case of $0<v<$ $v_{c 1}$ the probability density either at one or both boundaries $\left(x_{1}=y_{1}^{n}\right.$ and $\left.x_{2}=y_{2}^{n}\right)$ approaches infinity (see Figure 3 ), the corresponding singularities are integrable; that is, the probability distribution $\widetilde{P}(x)$ can be normalized. This follows from (25) and (27) using the fact that the quantities $A_{1}$ and $A_{2}$ are positive.

\section{Single Unidirectional Transitions}

In this section we consider the dependence of the mean population size $\langle X\rangle$ on the amplitude of environmental fluctuations. It is important to note that in a stationary regime the value of

$$
\langle X\rangle=\int_{x_{2}(a)}^{x_{1}(a)} x \widetilde{P}(x) d x
$$

depends strongly on the boundary points $x_{1}(a)$ and $x_{2}(a)$ for an attracting interval. If $\widetilde{K}_{0}<K_{0 c}<2 \widetilde{K}_{0}$ and the initial 


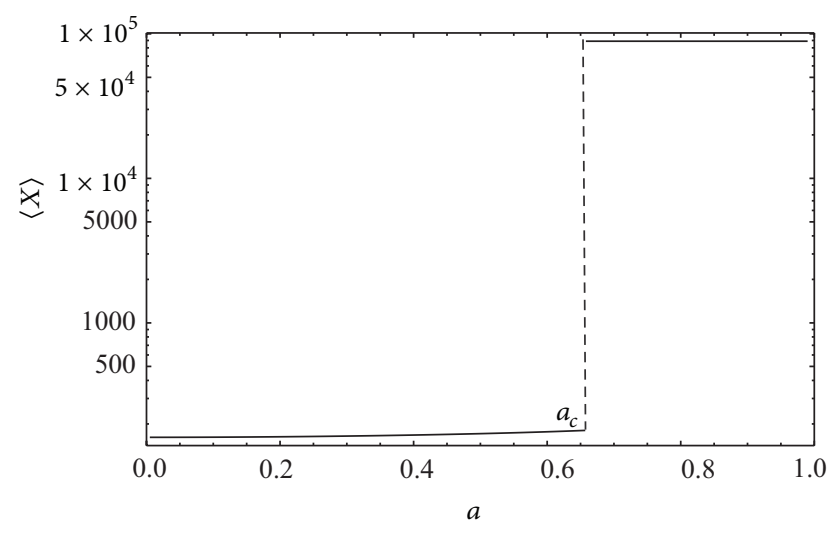

FIGURE 4: The stationary mean value of the population size $\langle X\rangle$ versus the noise amplitude $a$. The system parameter values are $\widetilde{K}_{0}=$ $100, \epsilon=0.03, \gamma=10^{-5}, \alpha=0.5$, and $\nu=75$. Note the jump of the mean population size $\langle X\rangle$ at the critical value of the noise amplitude, $a_{c} \approx 0.66$.

population size $x(0)$ is sufficiently small $\left(x(0) \leq \widetilde{K}_{0}\right)$, then by increasing the noise amplitude $a$ the stochastic population growth model ((5)-(9)) predicts the following scenario. For small values of the noise amplitude the mean population size grows in time and saturates finally at a value determined by the attracting interval (18). As $a$ increases the mean population size slowly increases, but as the noise amplitude approaches the critical value $a_{c}, K_{01}\left(a_{c}\right)=K_{0 c}$, the attracting interval (18) disappears and the system undergoes a transition to the state characterized by the attracting interval (17), where the population size is very much larger than in the interval (18). The further increase of $a$ causes a monotonic and very slow decrease of $\langle X\rangle$ (cf. Figure 4).

We emphasize that although an increase of the noise amplitude $a$ causes an abrupt transition from a stationary state of a lesser number of individuals to one of a very much bigger number, by a decrease of $a$ the opposite transition cannot occur. Let us look at the latter statement more closely on the assumption that at $a>a_{c}$ the initial population size $x(0)$ is in the attracting interval (17). The population size evolves by the influence of an "effective flipping potential," where the effective potential switches between two configurations $U_{\text {ef }_{i}}$ (cf. Figure 5 and also Figure 2)

$$
U_{\mathrm{ef}_{i}}(x)=-r \gamma \epsilon \int^{x} \frac{\xi F_{i}\left(\xi^{1 / n}\right)}{1+\gamma \epsilon \xi^{1+\alpha}} d \xi, \quad i=1,2 .
$$

In the case of $a>a_{c}$ one potential configuration is monostable and the other is bistable (see Figure 5(a)) and the values of $x$ are locked in the interval $\left(x_{2}, x_{1}\right)$, that is, in the attracting interval (17). As the noise amplitude $a$ decreases the monostable potential profile acquires a bistable profile at $a \leq a_{c}$, as a result of which the effective potential switches between two bistable configurations (see Figure 5(b)). But also in this case the population size $x$ remains locked between the positions $x_{12}$ and $x_{22}$ of the minima of the configurations 1 and 2 , respectively (i.e., in interval (19)). A similar phenomenon, called noise-induced single unidirectional transition, was previously considered in the context of symbiotic ecosystems [26], but the mechanism of that phenomenon is of a qualitatively different nature from the effect presented here.

\section{Conclusions}

Inspired by the Von Foerster growth model for human population [4], a generalization of the logistic growth model with a population size-dependent carrying capacity is given. Random influence of the environment on the dynamics of the population is taken into account by introducing a dichotomous noise in the carrying capacity. The exact expressions for the stationary probability density $\widetilde{P}(x)$ of population sizes $x$ are derived. One of our major results is the establishment of noise-induced transitions between three qualitatively different forms of a stationary population size distribution. At small noise correlation times the stationary distribution is characterized with a bell-shaped form, but as the correlation time of environmental fluctuations increases the system undergoes a transition to a state characterized with a probability density with either two or no local extrema, while a further increase of the correlation time brings another transition-the system goes to a state where the probability density exhibits a U-shaped form. Another result, perhaps the most important in the ecological context, is the existence of noise-induced single unidirectional transition for the mean population size (i.e., an increase in noise amplitude can cause an explosive growth of the size of population, while by decreasing the noise amplitude no opposite transitions can occur). It should be noted that noise-induced unidirectional transitions have been previously considered by investigations of symbiotic ecosystems [26], where an increase in noise amplitude can cause a catastrophic fall in the size of populations, but the mechanisms of those phenomena in [26] and in the current paper are qualitatively different.

Finally, we believe that the model and the results discussed here can be useful to describe the long-term behavior of the human population along the lines considered in [4$7,30]$ and can also be applied to other types of processes that are explosive in their nature due to a positive feedback. A further detailed study is, however, necessary, especially an investigation of transient processes. Our exact results obtained in the case of dichotomous noise can be a good starting point for investigations, by numerical simulations, of the dynamics of the population size in the presence of more realistic models for environmental colored noise.

\section{Conflict of Interests}

The authors declare that there is no conflict of interests regarding the publication of this paper. 


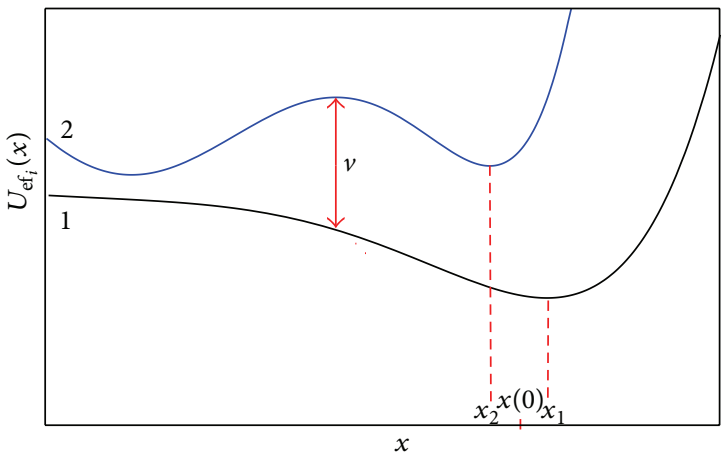

(a)

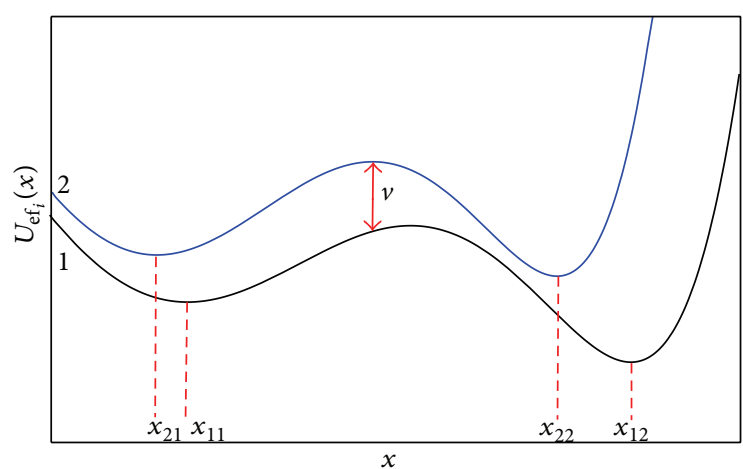

(b)

FIGURE 5: A schematic illustration of the effective potential configurations $U_{\mathrm{ef}_{i}}$ versus the population size $x, i=1,2$. Because of the influence of the noise $Z(t)$ the effective potential fluctuates with a switching rate $v$ between two configurations $U_{\mathrm{ef}_{1}}(x)$ and $U_{\mathrm{ef}}(x)$, which correspond to the noise values $z=1$ and $z=-1$ and are illustrated by curves 1 and 2, respectively. (a) The case of $a>a_{c}$; the positions of the minima (stable fixed points of (1) with (3)) of $U_{\mathrm{ef}_{1}}(x)$ and $U_{\mathrm{ef}_{2}}(x)$ are denoted by $x_{1}$ and $x_{2}$, respectively. (b) The case of $a<a_{c}$; the stable fixed points by the noise states $z=1$ and $z=-1$ are $x_{1 j}$ and $x_{2 j}, j=1,2$, respectively. The initial population size is denoted by $x(0)$ in (a).

\section{Acknowledgments}

The work was supported by the Estonian Science Foundation under Grant no. 9005, by the Ministry of Education and Research of Estonia under Grant no. SF0130010s12, by the International Atomic Energy Agency under Grant no. 14797, and by European Union through the European Regional Development Fund (Centre of Excellence "Mesosystems: Theory and Applications," TK114).

\section{References}

[1] P. Turchin, Complex Population Dynamics: A Theoretical/Empirical Synthesis, vol. 35, Princeton University Press, Princeton, NJ, USA, 2003.

[2] J. D. Murray, Mathematical Biology, vol. 19, Springer, Berlin, Germany, 1989.

[3] J. M. McGlade, Advanced Ecological Theory: Principles and Applications, Blackwell Science, Oxford, UK, 1999.

[4] H. Von Foerster, P. M. Mora, and L. W. Amiot, "Doomsday: Friday, 13 November, A.D. 2026," Science, vol. 132, no. 3436, pp. 1291-1295, 1960.

[5] A. Johansen and D. Sornette, "Finite-time singularity in the dynamics of the world population, economic and financial indices," Physica A, vol. 294, no. 3-4, pp. 465-502, 2001.

[6] D. Strzałka, "Connections between von Foerster coalition growth model and Tsallis q-exponential," Acta Physica Polonica $B$, vol. 40, no. 1, pp. 41-47, 2009.

[7] J. E. Cohen, "Population growth and earth's human carrying capacity," Science, vol. 269, no. 5222, pp. 341-346, 1995.

[8] O. N. Bjørnstad and B. T. Grenfell, "Noisy clockwork: time series analysis of population fluctuations in animals," Science, vol. 293, no. 5530, pp. 638-643, 2001.

[9] J. Laakso, V. Kaitala, and E. Ranta, "Population dynamic consequences of adaptive growth rate in fluctuating environment," Ecological Modelling, vol. 194, no. 1-3, pp. 132-140, 2006.

[10] R. Benzi, A. Sutera, and A. Vulpiani, "The mechanism of stochastic resonance," Journal of Physics A, vol. 14, no. 11, pp. L453-L457, 1981.
[11] K. Laas, R. Mankin, and A. Rekker, "Constructive influence of noise flatness and friction on the resonant behavior of a harmonic oscillator with fluctuating frequency," Physical Review E, vol. 79, no. 5, Article ID 051128, 2009.

[12] L. Gammaitoni, P. Hänggi, P. Jung, and F. Marchesoni, "Stochastic resonance," Reviews of Modern Physics, vol. 70, no. 1, pp. 223 287, 1998.

[13] R. Mankin, K. Laas, T. Laas, and E. Reiter, "Stochastic multiresonance and correlation-time-controlled stability for a harmonic oscillator with fluctuating frequency," Physical Review E, vol. 78, no. 3, Article ID 031120, 2008.

[14] R. Mankin and A. Rekker, "Memory-enhanced energetic stability for a fractional oscillator with fluctuating frequency," Physical Review E, vol. 81, no. 4, Article ID 041122, 2010.

[15] P. Reimann, "Brownian motors: noisy transport far from equilibrium," Physics Report, vol. 361, no. 2-4, pp. 57-265, 2002.

[16] P. Hänggi and F. Marchesoni, "Artificial Brownian motors: controlling transport on the nanoscale," Reviews of Modern Physics, vol. 81, no. 1, pp. 387-442, 2009.

[17] J. García-Ojalvo and J. M. Sancho, Noise in Spatially Extended Systems, Springer, New York, NY, USA, 1999.

[18] F. Sagués, J. M. Sancho, and J. García-Ojalvo, "Spatiotemporal order out of noise," Reviews of Modern Physics, vol. 79, no. 3, pp. 829-882, 2007.

[19] C. van den Broeck, J. M. R. Parrondo, R. Toral, and R. Kawai, "Nonequilibrium phase transitions induced by multiplicative noise," Physical Review E, vol. 55, no. 4, pp. 4084-4094, 1997.

[20] S. Mangioni, R. Deza, H. S. Wio, and R. Toral, "Disordering effects of color in nonequilibrium phase transitions induced by multiplicative noise," Physical Review Letters, vol. 79, no. 13, pp. 2389-2393, 1997.

[21] P. Hänggi, F. Marchesoni, and P. Grigolini, "Bistable flow driven by coloured gaussian noise: a critical study," Zeitschrift für Physik B Condensed Matter, vol. 56, no. 4, pp. 333-339, 1984.

[22] W. Horsthemke and R. Lefever, Noise-Induced Transitions, vol. 15, Springer, Berlin, Germany, 1984.

[23] E. Soika, R. Mankin, and A. Ainsaar, "Resonant behavior of a fractional oscillator with fluctuating frequency," Physical Review E, vol. 81, no. 1, Article ID 011141, 2010. 
[24] R. Mankin, K. Laas, and A. Sauga, "Generalized Langevin equation with multiplicative noise: temporal behavior of the autocorrelation functions," Physical Review E, vol. 83, no. 6, Article ID 061131, 2011.

[25] R. Mankin, K. Laas, and N. Lumi, "Memory effects for a trapped Brownian particle in viscoelastic shear flows," Physical Review E, vol. 88, no. 4, Article ID 042142, 2013.

[26] A. Sauga and R. Mankin, "Addendum to 'colored-noise-induced discontinuous transitions in symbiotic ecosystems," Physical Review E, vol. 71, no. 6, Article ID 062103, 2005.

[27] S. Kim, S. H. Park, and C. S. Ryu, "Colored-noise-induced multistability in nonequilibrium phase transitions," Physical Review E, vol. 58, no. 6, pp. 7994-7997, 1998.

[28] R. Müller, K. Lippert, A. Kühnel, and U. Behn, "First-order nonequilibrium phase transition in a spatially extended system," Physical Review E, vol. 56, no. 3, pp. 2658-2662, 1997.

[29] V. J. Klyatskin, Lectures on Dynamics of Stochastic Systems, Elsevier, London, UK, 2011.

[30] B. C. T. Cabella, F. Ribeiro, and A. S. Martinez, "Effective carrying capacity and analytical solution of a particular case of the Richards-like two-species population dynamics model," Physica A, vol. 391, no. 4, pp. 1281-1286, 2012. 


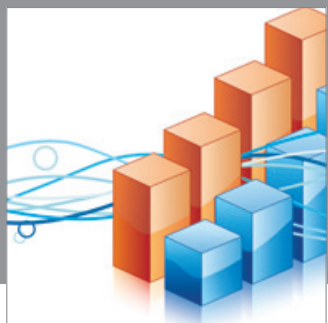

Advances in

Operations Research

mansans

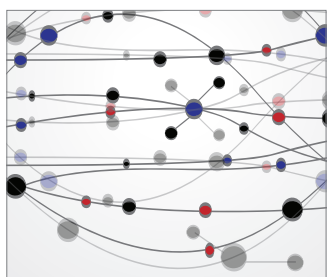

The Scientific World Journal
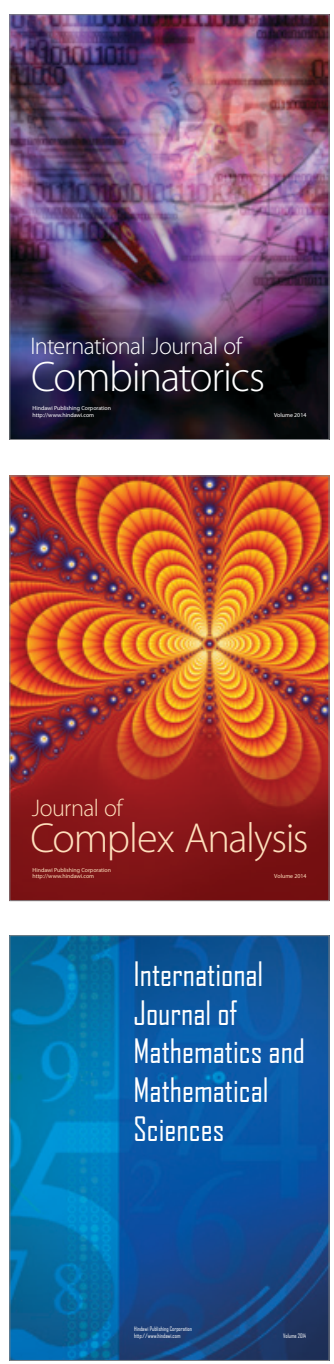
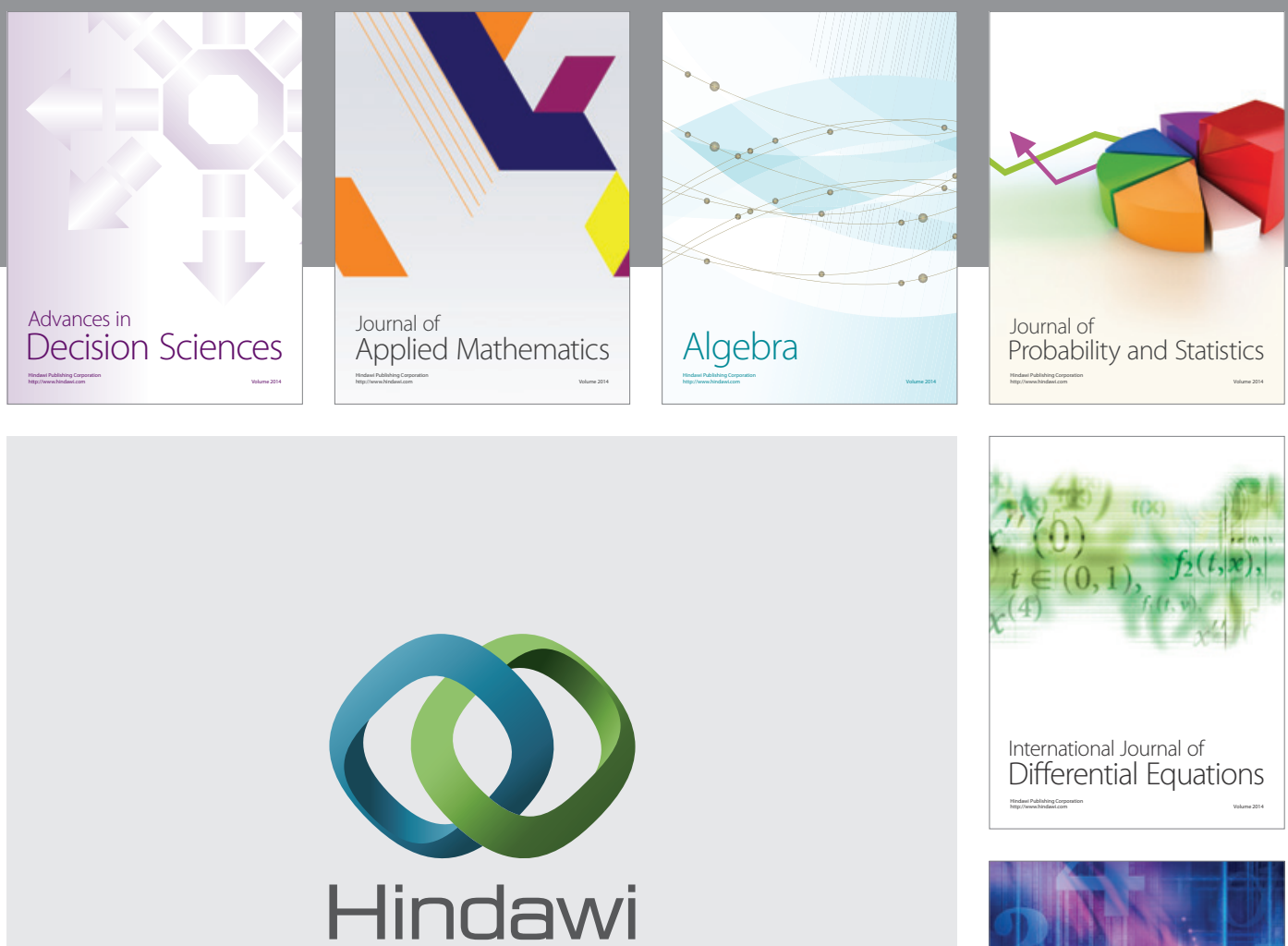

Submit your manuscripts at http://www.hindawi.com
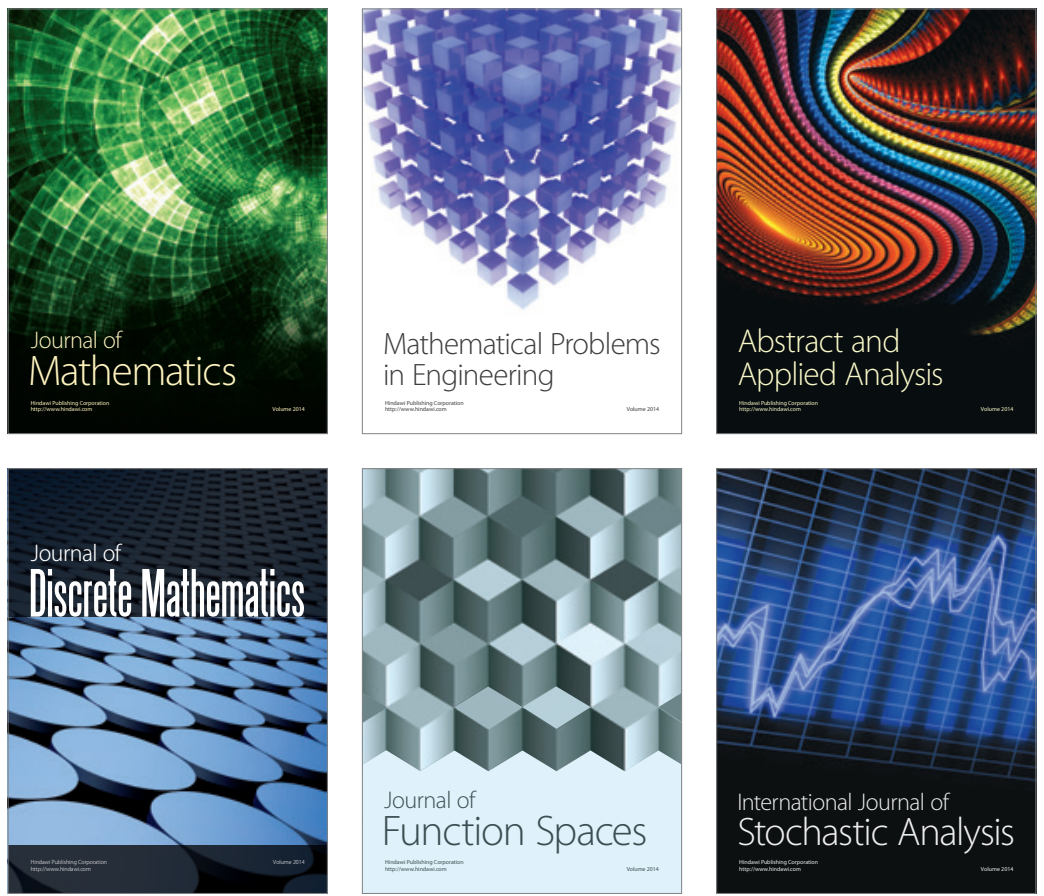

Journal of

Function Spaces

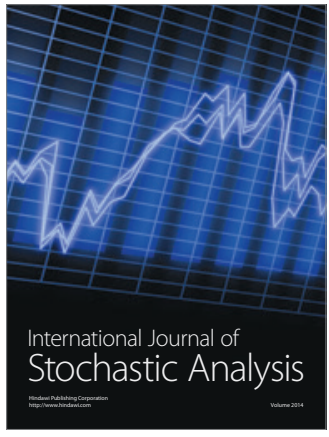

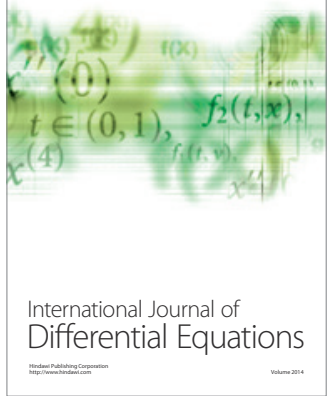
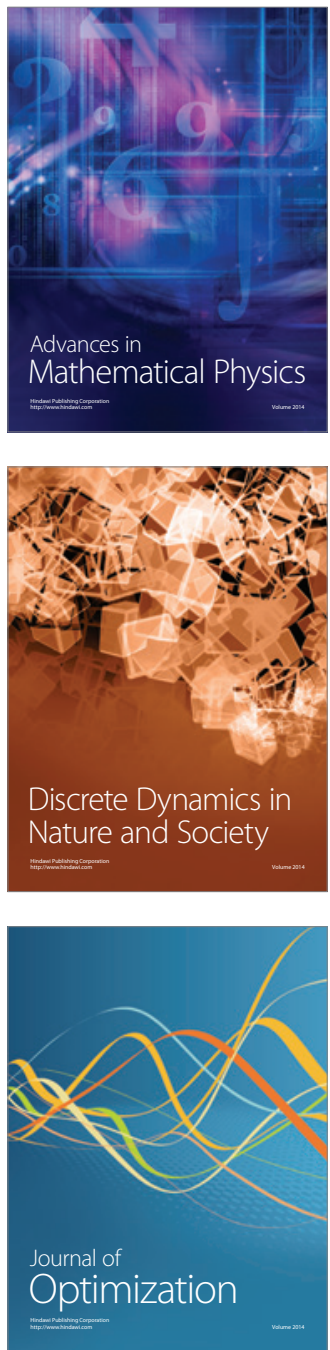